\title{
UNA IMAGEN REPRESENTATIVA DE PODER EN CENTROEUROPA A FINALES DEL SIGLO XVI
}

En el Museu de la Pell de Vic, se encuentra un pequeño cuadrito en el que se representa un retrato ecuestre de un caballero con armadura y banda, con la cabeza de marfil tallado sobre un gran caballo en cuero modelado en actitud de paseo. La figura destaca potentemente sobre un fondo dorado, en el que se advierte en su parte inferior un diminuto ejército en un campo de batalla, casi desaparecido ${ }^{1}$ (Fig.1). El trabajo utilizado, el modelado y repujado del cuero en la figura y en el caballo, se ve animado por el contraste de colores de la banda roja que ondea al viento, el marfil de la cabeza y el fondo dorado con densas nubes en su parte alta. Los rasgos peculiares del rostro del caballero nos hicieron pensar en un primer momento en la figura del emperador Rodolfo II, por su gran parecido con una medalla conmemorativa del emperador con el reverso con el globo terráqueo y el signo de Capricornio, realizada en los primeros años del siglo XVII, (Fig 4) ${ }^{2}$. Posteriormente constatamos que, en realidad, se trata de un retrato de su hermano, el archiduque Maximiliano III (Fig. 5), nombrado por Rodolfo gobernador del Tirol y Gran Maestre de la Orden Teutónica desde $1585^{3 *}$. Regente de la Austria Interior, desde 1593 a 1595 desarrolló una intensa actividad en el Tirol. Poco dado a la ostentación cortesana, se preocupo de la instrucción pública haciendo de Innsbruck un centro cultural relevante. Educado como sus hermanos en los valores estéticos de la Corte de los Habsburgo, dio preferencia en Innsbruck a los libros y a la música, creando la cámara del tesoro de la Wallfahrtkirche Mariastein bajo la tutela archiducal y sobre todo fundando la abadía de Klosterneuburg como símbolo de la unidad dinástica archiducal austriaca. Bajo su custodia como regente del Tirol estuvo desde 1602 la Kunstkammer de Ambras. Entusiasta defensor de la Contrarreforma, desde su cargo de Gran Maestre de la Orden alemana, tuvo la habilidad de conciliar también a los príncipes protestantes en la defensa contra las invasiones turcas desde 1594 a 1599 en Croacia y Alta Hungría, actuando como general por designación imperial, con desigual suerte.

Precisamente por su cargo de Gran Maestre de la Orden, su imagen pública debía continuar la tradición icónica seguida con éxito en la iconografía imperial centroeuropea: caballero con armadura y banda, imagen fijada en el retrato ecuestre de Rodolfo II realizado por Juan de Bolonia en 1595 con ligeras variantes: mayor movimiento de la cola del caballo y de la banda al viento, siguiendo los dibujos que Adrian de Vries realizó sobre el modelo del italiano durante los años siguientes en Praga ${ }^{4}$; en actitud triunfante, pero con la espada envainada; dotado el rostro de gran fuerza, lo que junto a la elevación orgullosa de la cabeza y la mirada en la lejanía, lo convirtió en un retrato de carácter enérgico y heroico, sobre todo en el retrato ecuestre del Museo de Estocolmo ${ }^{5}$ (Fig. 2), en una retórica del retrato imperial derivado en última instancia del Carlos V en Mühlberg. En el caso de Maximiliano se reproduce la iconografía principesca y la imagen sobreentendida de la época, en una actitud triunfante quizá fruto de las victorias de Patrina y Sissek en 1593, aunque fue después derrotado por los turcos en 1596 en la batalla de Erlau.

\footnotetext{
1 Museu de la Pell, Vic. Inventario nº 680, C/1999.

${ }^{2}$ Viena. Kunsthistorisches Museum. Gabinete de Medallas no 860 b. Catálogo Prag um1600 nº 476.

${ }^{3}$ Felipe II había llamado a Maximiliano a España para encargarle la sucesión de Alberto en el virreinato de Portugal, pero no llegó a venir. En la década de los noventa Maximiliano y Rodolfo recibían frecuentemente envíos de objetos de su madre María de Austria desde España, especialmente cajas de guantes fabricados aquí, según se registran en las cedulas de paso. Asimismo son frecuentes los regalos que desde Bruselas le enviaba su hermano, el archiduque Alberto. Véase Pérez de Tudela y Jordan, «Luxury goods for royal collectors» en Exotica.. Portugal Entdeckungen im Spiegel fürstlicher Kunst- und Wunderkammern derRrenaissance. Jahrbuch des Kunsthistorischen Museums Wien. 3 .2001. Appendix A.

${ }^{4}$ Como el conservado en el museo Paul Getty de Malibú, realizó numerosas interpretaciones y copias como objetos de colección de los que en diversos tamaños y posiciones había en el taller de Juan de Bolonia, muchas pertenecientes a entonces a las colecciones de Rodolfo II.

${ }^{5}$ Nationalmuseum de Estocolmo Inv $\mathrm{n}^{\circ} 749$, procedente de la colección de Cristina de Suecia desde 1652
}

AEA, LXXVIII, 2005, 309, pp. 83 a 105 

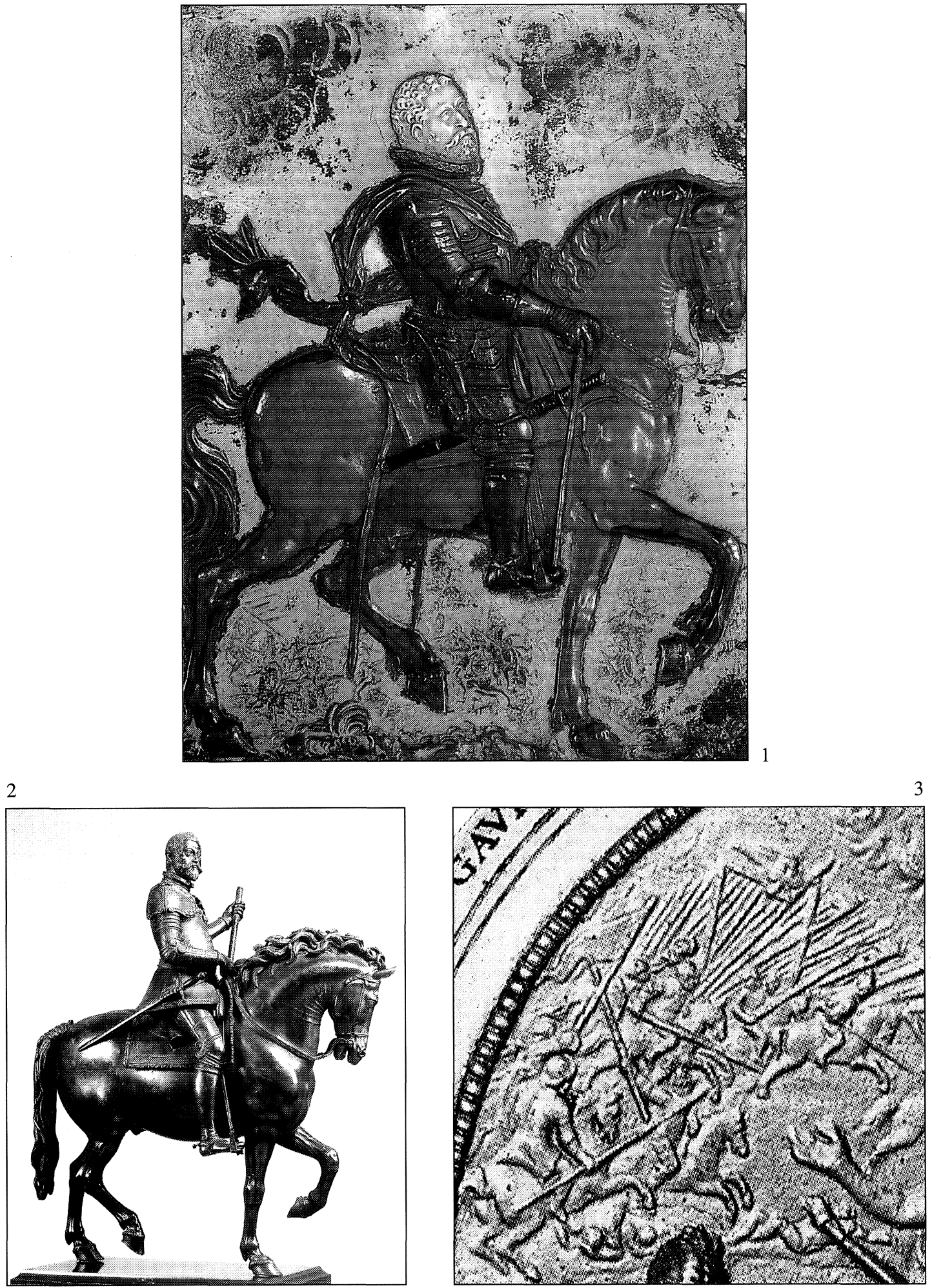

Fig. 1. Maximiliano III, archiduque de Austria. Marfil y piel. Vic. Museu de la Pell.

Fig. 2. Juan de Bolonia: El emperador Rodolfo II. 1595. Bronce. Estocolmo. Museo Nacional.

Fig. 3. Paulus van Vianen: Medalla conmemorativa de la toma de Tergowist. 1595. Detalle. Viena Kunsthistorischesmuseum. Münzenkabinett. 
El cuadro reproduce con exactitud una placa de plata atribuida a Jan de Vos, conservada en Viena en la Schatzkammer de la Orden ${ }^{6}$ (Fig. 6). Esta atribución al platero de Augsburgo se basa en el testimonio de Philipp Hainhofer, identificando el retrato ecuestre del archiduque realizado por Hubert Gerhard y su transformación en relieve por el augsburgués, quien fue también platero de Rodolfo II. En Praga aprendió y siguió en estilo puesto de moda por Paulus van Vianen, el destacado platero de Utrecht, también llamado a la corte imperial, especializado en el repujado en plata y oro, que depuró está técnica de modo inigualable, consiguiendo efectos pictóricos como ningún platero antes e influyendo de una forma decisiva sobre el barroco neerlandés ${ }^{7}$. Tanto en la ciudad bohemia como en Augsburgo tuvo estrecha relación con Adrian de Vries, quien en 1594 estuvo también en Augsburgo, volviendo a Praga como escultor de cámara del emperador en 1601. La poderosa figura del caballo tomó carta de naturaleza en los bronces de pequeño formato del Renacimiento italiano sobre todo a partir del realizado por Juan de Bolonia para el retrato ecuestre de Cosme de Medicis, inspirándose en la estatua de Marco Aurelio, figuras en las que se pone de manifiesto la «estabilidad y autoridad del poder» del personaje representado. El ejemplo más cercano en pequeño formato en la corte imperial es sin duda el realizado por Antonio Susini, que se levanta sobre un pedestal de piedras duras perteneciente al taller de los Castrucci, célebres comettitori florentinos, que desarrollaron su actividad en la corte rudolfina, si bien el modelo fue sin duda el que Adriaen de Vries realizó para Rodolfo II, que permaneció en la Kunstkammer imperial, hasta su traslado a Suecia en la guerra de los Treinta Años.

La escena de batalla en miniatura que se deja ver entre las patas del caballo, está resuelta en tres grupos de soldados a caballo en actitud de marcha hacia la derecha, otro grupo al centro les hace frente un caballero con lanza y escudo, mientras que a la derecha se representa un grupo de soldados a pie también con escudos y lanzas. Este tratamiento en miniatura está copiado de una medalla, atribuida igualmente a Paulus van Vianen que conmemora la toma de Tergowist, capital de Siebengebürgen por Segismundo Báthory en 1595, representando a Bellona con el cetro en una isla en el río, en el que flotan cabezas de turcos y ante ella los rios Save y Danubio, representándose a la izquierda una escena de batalla, como la de nuestra placa (Fig. 3) y a la derecha una ciudad; en el reverso de la misma se conmemora la reconquista de Roah en 1598 por Adolf von Schwarzenberg ${ }^{8}$. Estas series de medallas conmemorativas de las victorias contra los turcos se realizaron siguiendo los modelos de las Inventiones de Hans von Aachen, que asimismo sirvieron de modelo a Adrian de Vries para su relieve con la Alegoría de la guerra de Hungría de 1603, exactamente la misma fecha en que Paulus van Vianen inicia su estancia en Praga. La utilización de los fondos de batallas resalta el carácter propagandístico de legitimación del poder, trasladándose a pinturas y tapicerías de gran formato sobre todo en el segundo tercio del siglo XVII ${ }^{9}$.

Parece que Jan de Vos, estuvo especializado en estas piezas de pequeño formato, piezas destinadas precisamente a formar parte de las colecciones de las Kunstkammern, a las que se

\footnotetext{
${ }^{6}$ Wien Schatzkammer des Deutschen Ordens, Inv. № 114. Exposición Prag um 1600. Cat. № 537. Para su figura, H.Noflatscher «Glaube, Reich und Dynastie: Maximilian der Deutschmeister (1558-1618)» en Quellen und Studien zur Geschichte des Deutschen Ordens. 1987.

${ }^{7}$ Del taller de Juan de Bolonia proceden otros retratos ecuestres en pequeño formato con el mismo modelo. Uno en la colección Liechtenstein con el retrato de Fernando I y otro en la Wallace Collection, representando esta vez a Enrique IV de Francia. Vid. Ch. Avery, Giambologna. The compete sculpture. Oxford 1987; Adiaen de Vries (1556-1626). Augsburgs Glanz-Europas Ruhm. Augsburg 2000., Cat. N 18.

${ }^{8}$ Kunsthistorischenmuseum de Viena. Münzenkabinett, Inventario n ${ }^{\circ} 67 \mathrm{bb}$. Formó parte de la exposición Prag um 1600. Kunst und Kultur am Hofe Rudolf II. Wien 1988-1999 cat $\mathrm{n}^{\circ} 467$

${ }^{9}$ Pfaffenbichler, M. «Das barocke Schlachtenbild- Versich einer Typologie». Jahrbuch des Kunsthistorischen Sammlungen in Wien. 91. 1995. 37-110.
}

$A E A$, LXXVIII, 2005, 309, pp. 83 a 105 

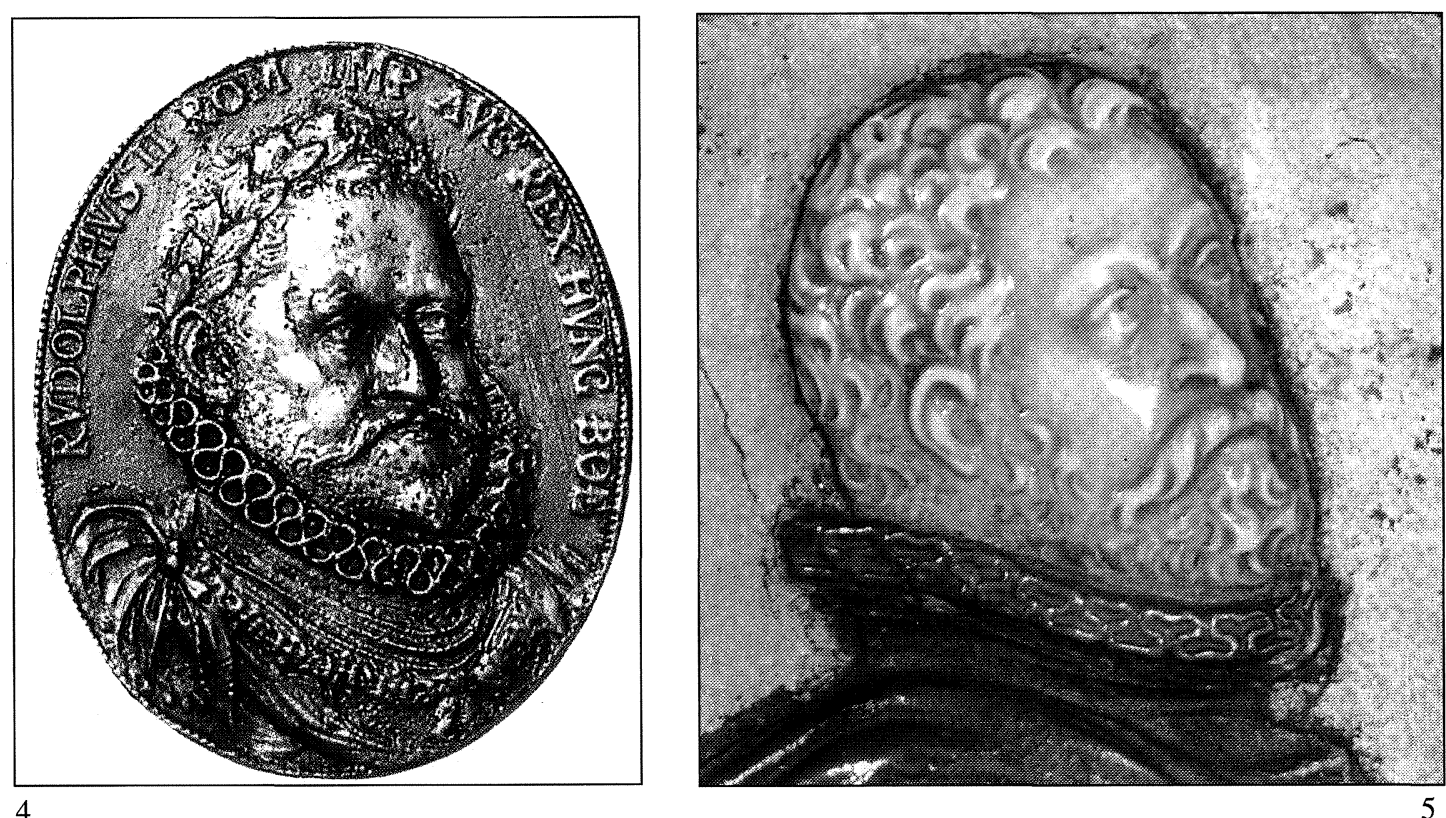

6
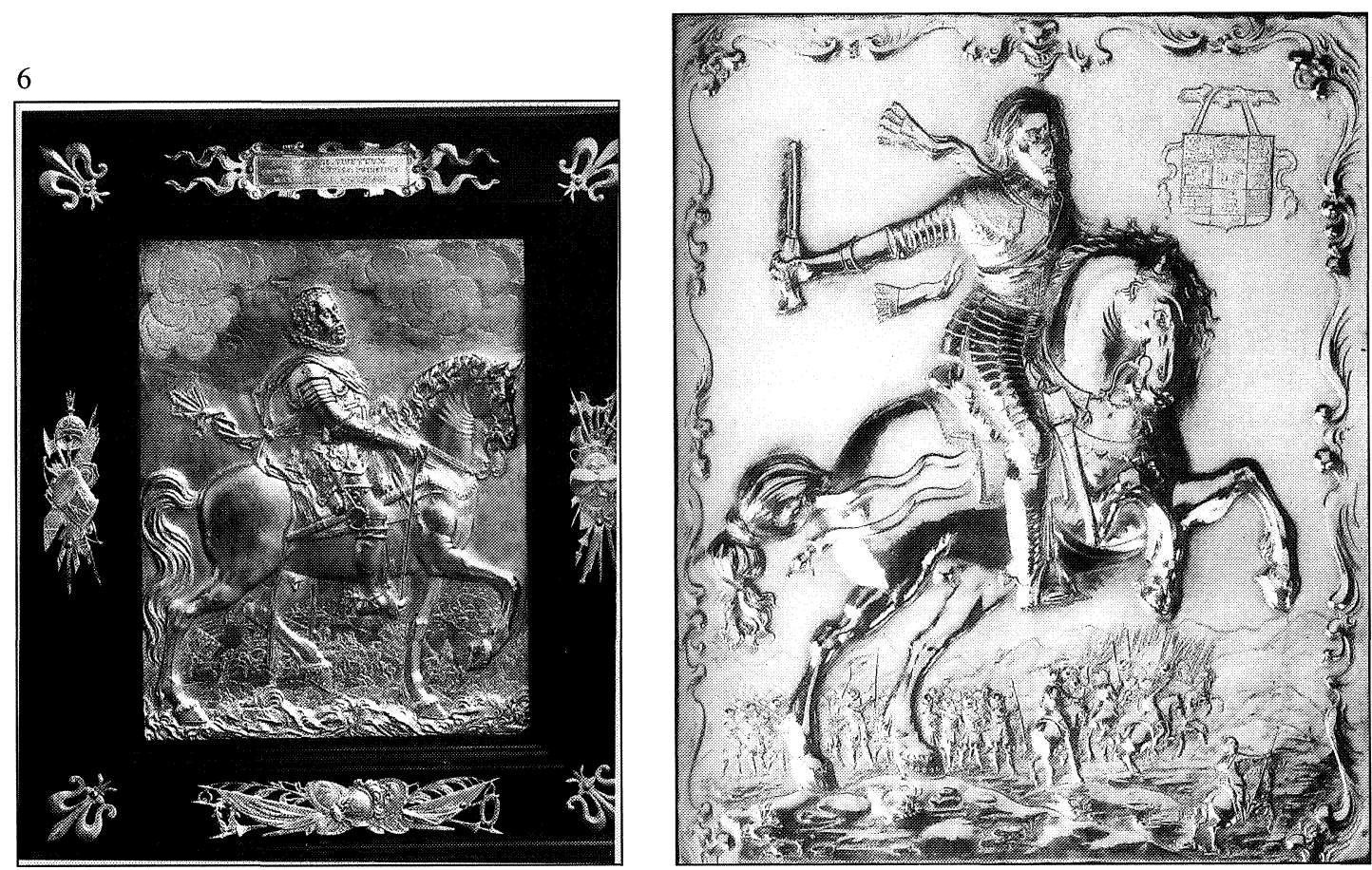

Fig. 4. Medalla de Rodolfo II, h. 1600. Viena Kunsthistorischesmuseum. Münzenkabinett.

Fig. 5. Archiduque Maximiliano III (detalle). Marfil. Vic. Museu de la Pell.

Fig. 6. Atribuida a Jan de Vos: Maximiliano II como gran Maestre de la Orden Teutónica. Plata y ébano, h. 1600. Viena. Schatzkammer des Deutschen Ordens.

Fig. 7. Retrato ecuestre de Christian, duque de Braunschweig-Luneburg-Wolfenbüttel el Joven, h. 1620. Plata. Utrecht. Mercado de Arte. 
dedicaron con interés los plateros de Augsburgo ${ }^{10}$, donde realizó varias réplicas de la de Maximiliano III, a cuyo servicio estuvo antes de su vuelta definitiva a Augsburgo. Según relata Hainhofer realizó una versión en cera de la estatuilla de Hubert Gerhard, conservándose uno de los relieves en plata en la colección de los príncipes de Liechtenstein en Vaduz y se cree que la invención del relieve correspondió en esencia más a Huber Gerhard que al propio Vos. En el último cuarto del siglo xIx la firma C.Heas de Viena realizó destacadas reproducciones galvanoplásticas a partir de este relieve del tesoro vienés, que pasaron al mercado del arte, conservándose algunas en museos como el Kunsthistorische Museum de Viena y el Museum für Kunst und Kunsthandwerk de Dortmund. Existen también réplicas en bronce en Viena, en el Bayerische Nationalmuseum de Munich y en las colecciones de Klosterneuburg, fundación del propio archiduque, todas ellas de indudable antigüedad. Posiblemente este pequeño cuadro realizado en cuero y marfil responde a esa difusión del modelo, realizado en unos materiales muy del gusto de principios del siglo xviI, como fueron el cuero y el marfil, material que, por cierto también trabajó Jan de Vos ${ }^{11}$.

En el museo Lázaro Galdiano se conserva un retrato ecuestre de bulto en bronce que se ha identificado como retrato también de un archiduque austriaco y que, por el tipo de armadura y peinado, pertenece a una fecha un poco posterior, ya de la segunda década del siglo XvII. Coppel remite a idénticos modelos iconográficos para el caballo, que los aquí citados y presenta como posible autor de la escultura a Caspar Gras (1584/85-1674), a quien se atribuye un grupo de jinetes semejantes en el Kunsthistorisches Museum de Viena que representan personajes de la familia imperial austriaca. Gras fue como Jan de Vos, discípulo de Hubert Gerard en Innsbruck, en cuya catedral y se encargo de terminar precisamente la tumba de nuestro Maximiliano III ${ }^{12}$.

El gusto por este tipo de objetos de pequeño tamaño, especialmente las placas de plata persistió al menos un cuarto de siglo en Europa como muestra el retrato ecuestre de Christian duque de Braunschweig Luneburg-Wolfenbüttel el Joven, también con armadura, aunque con diferente posición del caballo, que denota una fecha más avanzada, realizada en Utrecht hacia 1620 , por un platero cercano a los Van Vianen ${ }^{13}$, sobre un fondo casi idéntico, con la misma disposición de un ejército en el campo de batalla, casi imperceptible en su lejanía (Fig. 7). De este modo queda patente la continuación de la estrecha relación de estos plateros de Utrecht con la corte de Praga, con la que asimismo estuvo muy relacionado el primer duque de Braunschweig, quien conoció las obras citadas y encargó allí numerosas obras de arte, una muestra más de la interactuación de los círculos artísticos europeos durante la Edad Moderna.

María Paz Aguiló Instituto de Historia. CSIC

\footnotetext{
${ }^{10}$ Para Jan de Vos véase Silber un Gold. Augsburgischer Goldschmiedekunst für die Höfe Europas.. Hirmer. Munich 1994. 302-304.

11 Habich, Georg. «Jan de Vos oder Paulus van Vianen?» Das Schwäbische Museum, 1927, 124-135.

${ }^{12}$ R.Coppel Aréizaga Pequeños bronces en la Fundación Lázaeo Galdiano. Siglos XVI-XIX. Madrid 2001. pp.77-78

${ }^{13}$ Christie's Amsterdam, Abril 2002, lote 1.126
} 